\title{
O cuidado em enfermagem a pessoas idosas dependentes: cuidados domiciliares, hospitalares e continuados
}

\author{
Nursing care of dependent aged persons: home, hospital and continuing care
}

Atención de enfermería a ancianos dependientes: cuidados domiciliarios, hospitalarios y permanentes

Liliana Xavier Marques de Sousa ${ }^{1}$, Maribel Domingues Carvalhais², Lucibel Domingues Carvalhais ${ }^{3}$

\section{RESUMO}

O estudo objetivou identificar e comparar nos contextos da atenção básica, secundária e de reabilitação os cuidados de enfermagem prestados aos pacientes idosos dependentes, identificando os fatores que facilitam e dificultam a prestação desses cuidados de maior qualidade. Trata-se de pesquisa qualitativa realizada em serviços de saúde, que adotou o photovoice que usa fotografia e voz na coleta de dados. Participaram doze enfermeiros dos três contextos com idades entre os 22 e 43 anos, sendo dez do sexo feminino. Os resultados sugerem que a promoção da qualidade de cuidado em enfermagem a pessoas idosas dependentes envolve mais trabalho e decisão em equipe multidisciplinar. Paralelamente, o principal obstáculo centra-se na escassez de recursos e desorganização. Os participantes salientam a importância do envolvimento da família, considerada parte da unidade de cuidado. Concluindo, os três contextos tendem a convergir em termos das necessidades identificadas para a promoção da qualidade dos cuidados.

Descritores: Cuidados de Enfermagem; Saúde do Idoso; Serviço Hospitalar de Admissão de Pacientes; Atenção Primária à Saúde; Casas para Recuperação.

\section{ABSTRACT}

The objective was to identify and compare the nursing care provided to dependent aged patients within the settings of primary, secondary, and rehabilitative care, and identify the aspects that facilitate or hamper the delivery of care. This qualitative study was performed in healthcare services, using photovoice, which uses photography and voice recording for data collection. The participants were twelve nurses from the three referred contexts, aged between 22 and 43 years, ten of whom were women. Results suggest that the promotion of quality nursing care of dependent aged persons involves more work and decisions made by a multidisciplinary team. The main obstacles are the scarcity of resources and disorganization. The participants emphasize the importance of involvement by the family, who are considered to be part of the care team. In conclusion, the three referred contexts tend to converge in terms of the needs identified for the promotion of quality care. Descriptors: Nursing Care; Health of the Elderly; Admitting Department, Hospital; Primary Health Care; Halfway Houses.

\section{RESUMEN}

Se objetivó identificar y comparar en contexto de atención básica, secundaria y de rehabilitación, la atención de enfermería brindada a pacientes ancianos dependientes, e identificar factores que facilitan y dificultan la prestación de cuidados de enfermería de mayor calidad. Investigación cualitativa realizada en servicios de salud, que adoptó el photovoice, que utiliza fotografía y voz en la recolección de datos. Participaron 12 enfermeros de los tres contextos, con edades entre 22 y 43 años, 10 de sexo femenino. Los resultados sugieren que la promoción de calidad de atención en enfermería a ancianos dependientes involucra más trabajo y decisión en equipo multidisciplinario. Paralelamente, el principal obstáculo se centra en la escasez de recursos y desorganización. Los participantes enfatizan la importancia de la participación familiar, considerada parte de la unidad de atención. Concluyendo, los tres contextos tienen a converger en términos de las necesidades identificadas para la promoción de calidad del cuidado.

Descriptores: Atención de Enfermería; Salud del Anciano; Servicio de Admisión en Hospital; Atención Primaria de Salud; Casas de Convalecencia.

\footnotetext{
${ }^{1}$ Doutora em Ciências da Saúde. Professora Auxiliar com Agregação, Universidade de Aveiro. Aveiro, Portugal. E-mail: lilianax@ua.pt.

2 Doutora em Ciências da Saúde. Professora Adjunta, Escola Superior de Enfermagem Cruz Vermelha Portuguesa de Oliveira de Azeméis. Oliveira de Azeméis, Portugal. E-mail: mari.carvalhais@sapo.net.

${ }^{3}$ Enfermeira. Centro Hospitalar Baixo Vouga, Serviço de Oncologia. Aveiro, Portugal. E-mail: lucicarvalhais@hotmail.com.
} 


\section{INTRODUÇÃO}

As pessoas idosas apresentam mais doenças crônicas, comorbidades e índices superiores de dependência. Por isso, são os usuários mais comuns dos três níveis de cuidados de saúde: centro de saúde (cuidados primários - prevenção e promoção de saúde), hospital (secundários - tratamento e cura) e cuidados continuados (terciários - reabilitação e reintegração social).

Pesquisas ${ }^{(1-2)}$ sugerem que a qualidade dos cuidados de enfermagem a pessoas idosas tende a ser comprometida por duas razões: os enfermeiros prestam cuidados de forma mais apressada, dispensando o mínimo tempo necessário buscando justificações no cumprimento de rotinas impostas pela instituição e centram-se nos cuidados técnicos e na execução de rotinas, evitando cuidados mais expressivos. Além disso, as pessoas idosas são usuárias que, por norma, necessitam de mais cuidados e tempo (por comparação a pacientes mais novos), porque tendem a estar mais doentes e ser dependentes e/ou lentos na realização de atividades funcionais ${ }^{(3)}$. Por isso, tornam-se pacientes mais incómodos num contexto em que enfermeiros (e outros profissionais de saúde) têm muito trabalho e recebem diversas solicitações.

Principalmente, na vida das pessoas mais idosas dependentes os contextos (primário, secundário e terciário) de cuidados estão muito interligados e são utilizados de forma circular, por apresentarem complexidade de problemas de saúde.

Em Portugal, o apoio domiciliar é articulado aos cuidados de saúde primários, organizados nos centros de saúde e sucedem a alta hospitalar, pois as internações são cada vez mais curtas e os doentes, especialmente os mais velhos, desejam ir para casa rapidamente. Além disso, as pessoas idosas apresentam, principalmente, condições crônicas que exigem cuidados em casa ${ }^{(3-5)}$. A qualidade dos cuidados domiciliares depende da efetivação de cuidados técnicos, mas o processo de cuidar em enfermagem engloba também aspectos interpessoais e humanistas na relação entre enfermeiro, usuário e sua família(5). Tais aspectos podem comprometer ou favorecer os cuidados, em termos de evolução e efetividade, pois os usuários valorizam o fato de serem acolhidos, escutados e compreendidos pelos enfermeiros ${ }^{(5)}$.
A internação hospitalar também pode ocorrer em pessoas idosas que usufruem de cuidados domiciliares, pois são com frequência pessoas com comorbidades e elevada probabilidade de ocorrência de episódios agudos que podem levar à hospitalização e, até, à manutenção frequente deste circuito. $O$ incentivo à alta hospitalar precoce dos pacientes impõe desafios aos enfermeiros em contexto hospitalar, usuários e seus familiares. Os enfermeiros terão de preparar pacientes e famílias para reorganizarem a vida em casa no sentido de receber o paciente ainda em recuperação; o doente tem de se adaptar à nova condição; e os familiares têm de se organizar para assumir os cuidados necessários(6-7).

O prolongamento do período de internação também constitui um desafio (para além de aumentar os custos), principalmente pelo aumento dos riscos de complicações médicas e iatrogênicas ${ }^{(8)}$. A alta hospitalar coloca ainda o desafio do encaminhamento de pessoas idosas dependentes sem suporte familiar, cuja família não tem condições financeiras ou habitacionais para as receber, ou que precisam de trabalhar e não podem prestar cuidados $^{(7-8)}$. Nestes casos, os cuidados domiciliários (situações menos graves) ou os cuidados continuados (situações mais graves) serão a resposta, ponderada segundo a situação clínica do doente.

Quando há necessidade ou possibilidade de reabilitação, os doentes após alta hospitalar seguem para as unidades de cuidados continuados, onde ficam por períodos determinados de tempo (entre um mês a um ano). A rede de cuidados continuados foi recentemente criada em Portugal, para responder a necessidades atuais de saúde e sociais, incluindo satisfazer o incremento da procura por pessoas idosas dependentes que também necessitam de apoio psicossocial(9-10).

Neste contexto sublinha-se a importância da família, pois em Portugal a família é o centro da tradição da responsabilidade pela prestação de cuidados; contudo também tem de assumir os cuidados devido à escassez de serviços organizados(2).

Este estudo objetivou identificar e comparar nos contextos da atenção básica, secundária, e de reabilitação, os cuidados de enfermagem prestados aos pacientes idosos dependentes e identificar os fatores que facilitam e dificultam a prestação de cuidados de enfermagem de maior qualidade. Os resultados são relevantes para identificar fatores de promoção da 
qualidade dos cuidados a pessoas idosas dependentes em diversos contextos de saúde e para identificar modos de fomentar, sensibilizar e motivar os enfermeiros para a prestação de cuidados de maior qualidade.

\section{METODOLOGIA}

Estudo qualitativo realizado entre abril e setembro de 2008, tendo decorrido num serviço de medicina hospitalar (onde se concentram os cuidados às pessoas idosas); em três centros de saúde do distrito de Aveiro/Portugal; e numa unidade de cuidados continuados.

O recrutamento dos participantes foi realizado pela primeira autora, com procedimentos similares nas várias instituições, começando por uma breve apresentação do projeto aos enfermeiros chefes, indicando problema, objetivos, metodologia, participação solicitada e critérios de inclusão (ser enfermeiro, trabalhar no serviço há pelo menos seis meses e ter experiência profissional com pessoas idosas). Ao final deixava um resumo do projeto, juntamente com uma ficha de inscrição, solicitando a divulgação do pedido; cerca de uma semana depois recolhia as folhas de inscrição, tendo obtido 15 inscritos (seis enfermeiros de hospital, seis de centro de saúde e três de cuidados continuados). Realizou-se uma reunião individual com cada inscrito para explicar em detalhes o projeto e a colaboração solicitada. Os 15 que se disponibilizaram a participar, assinaram os consentimentos livres e informados. Contudo, três participantes de contexto hospitalar desistiram no decorrer das sessões, por indisponibilidade. Assim, a amostra envolve 12 enfermeiros (três enfermeiros de hospital, três de cuidados continuados e seis de centro de saúde) com idades entre os 22 e 43 anos, sendo 10 do sexo feminino.

A recolha dos dados utilizou o photovoice, um processo de investigação e intervenção participativo (qualitativo) em que os participantes identificam a sua vivência pela fotografia (imagem) e discutem em grupo (voz). É uma forma de aceder às realidades quotidianas dos participantes que são assumidos como especialistas nas suas experiências(11-12). Este método é flexível prevendo a adaptação a diversos tópicos de pesquisa e intervenção. Tem sido utilizado com diversas populações ${ }^{(11-13)}$, tais como refugiados, crianças de rua, sem-abrigo.

O photovoice desenvolve-se nas seguintes fases ${ }^{(11-12)}$ : a) conceptualização do problema (neste projeto centra a qualidade dos cuidados de enfermagem a pessoas idosas dependentes);

b) definição das finalidades e objetivos (neste caso foca os cuidados de enfermagem às pessoas idosas dependentes em contexto hospitalar, centro de saúde e cuidados continuados, e na reflexão sobre obstáculos e potencialidades para melhorar a sua qualidade);

c) selecionar e recrutar os participantes (foram recrutados enfermeiros que trabalham em serviço de medicina hospitalar, centros de saúde e cuidados continuados);

d) conduzir o Photovoice (trata-se da fase de desenvolvimento dos grupos, que envolveu três grupos, um em cada contexto, que se descreve no Quadro 1).

Quadro 1: Descrição das sessões de photovoice. Aveiro, Portugal, 2008.

\begin{tabular}{|c|l|}
\hline Sessão & \multicolumn{1}{|c|}{ Tópicos de discussão } \\
\hline 1 & $\begin{array}{l}\text { Apresentação dos membros do grupo e do projeto } \\
\text { Breve apresentação do Photovoice } \\
\text { Apresentação e discussão do problema } \\
\text { Introdução à questão 1 (O que é o cuidado em enfermagem?) }\end{array}$ \\
\hline \multirow{2}{*}{2 a 7} & $\begin{array}{l}\text { Apresentação das fotografias e discussão } \\
\text { Seleção pelo grupo da fotografia que melhor responde à questão e discussão } \\
\text { Introdução da questão seguinte } \\
\text { (Questão 2. O que é ser uma pessoa idosa dependente?) } \\
\text { (Questão 3. O que é o cuidado em enfermagem ideal a um idoso no contexto X?) } \\
\text { (Questão 4. O que é o cuidado em enfermagem real a um idoso no contexto X?) } \\
\text { (Questão 5. O que afasta o cuidado em enfermagem real do ideal?) } \\
\text { (Questão 6. O que aproximaria o cuidado em enfermagem real do ideal? }\end{array}$ \\
\hline 8 & $\begin{array}{l}\text { Apresentação de todas as fotografias e discussão sobre o problema inicial: "Como promover cuidados de enfermagem } \\
\text { de qualidade a pessoas idosas dependentes?" } \\
\text { Análise do impacto da participação no estudo. }\end{array}$ \\
\hline
\end{tabular}


Na primeira sessão são expostos os procedimentos éticos a usar na obtenção de fotografias, com destaque para o consentimento livre e informado. No período entre sessões, os participantes tiram as fotografias e antes da sessão seguinte selecionam três a quatro que considerem mais relevantes. Durante a sessão cada participante apresenta as suas fotografias e o grupo elege a fotografia que melhor traduz o pensamento do grupo.

As sessões tiveram um espaçamento entre uma e duas semanas e foram conduzidas pela primeira autora. Tiveram duração entre 60 e 120 minutos e todas foram gravadas com autorização dos participantes. Durante o processo foram sentidas algumas dificuldades, tais como: os participantes referiam dificuldades em retratar em fotografia a sua representação do tema; existia tendência para dispersar o discurso (por exemplo, com referências a vivências do serviço e/ou a casos que acompanham); a marcação das sessões era difícil, devido a necessidade de conciliar horários de participantes que fazem turnos e têm diversos part-times; as reuniões eram efetuadas após o trabalho, já estando todos um pouco cansados.

Todas as sessões foram gravadas, transcritas e submetidas a análise de conteúdo por três juízes independentes. Neste processo considerou-se a literatura e investigação na área da qualidade do cuidado em enfermagem e da dependência na velhice, mas procurou-se ser descritivo e manter, sempre que possível, o discurso dos participantes (mantendo as suas palavras e/ou expressões). Decidiu-se sempre que possível adotar as categorias sugeridas pelo grupo e partir das fotografias eleitas em cada sessão. O processo envolveu a construção de temas ${ }^{(14)}$ que traduzissem a opinião dos participantes. Com base num processo de codificação aberta, foram decompostas em unidades de análise, sendo critério de definição a representação de uma ideia única.

Foram adotados nomes fictícios aos participantes da pesquisa, os quais são identificados pelos mesmos e pelo local de trabalho.

Este estudo foi aprovado pela Comissão de Ética do Hospital Infante D. Pedro, Sub-Região de Saúde de Aveiro e Unidade de Cuidados Continuados de Barro.

\section{RESULTADOS}

O Quadro 2 apresenta os resultados emergentes das sessões de photovoice.

Quadro 2: Temas emergentes e contextos de cuidados. Aveiro, Portugal, 2008.

\begin{tabular}{|c|c|c|}
\hline Hospital & Centro de Saúde & Cuidados Continuados \\
\hline \multicolumn{3}{|c|}{ O que é o cuidado em enfermagem? } \\
\hline Mão & Presença & Mãos dadas \\
\hline Dialogar/comunicar & Dialogar/comunicar & Dialogar/comunicar \\
\hline Executar & Executar & \\
\hline Estar disponível & Estar disponível & \\
\hline Humanizar & & Humanizar \\
\hline \multicolumn{3}{|c|}{ O que é ser idoso dependente? } \\
\hline Necessitar de ajuda & Necessitar de apoio & Necessidade de readaptação \\
\hline Abandonado pela família & Abandonado & \\
\hline \multicolumn{3}{|c|}{ O que é o cuidado de enfermagem ideal a pessoas idosas dependentes? } \\
\hline \multirow[t]{2}{*}{ Satisfazer as necessidades } & Parcerias & Integra a família \\
\hline & "Ideal humano" & \\
\hline \multicolumn{3}{|c|}{ O que é o cuidado de enfermagem real a pessoas idosas dependentes? } \\
\hline Prestado no limite & Parcial & "Os que prestamos!" \\
\hline Falta de apoio da família & Abandono pela família & \\
\hline \multicolumn{3}{|c|}{ O que afasta os cuidados reais do ideal? } \\
\hline \multirow[t]{2}{*}{ Escassez e desorganização } & Escassez e desorganização & Não envolvimento da família \\
\hline & & Escassez \\
\hline \multicolumn{3}{|c|}{ O que facilitaria que o cuidado real em enfermagem se aproximasse do ideal? } \\
\hline Trabalho em equipa multidisciplinar & Manter o sonho (vontade) & Trabalho em equipa multidisciplinar \\
\hline \multirow[t]{2}{*}{ Material } & Material & Material \\
\hline & Equipa multidisciplinar & \\
\hline
\end{tabular}




\section{$1^{a}$ Sessão}

Centrou a pertinência do tema em estudo (promover cuidados de enfermagem de qualidade a pessoas idosas dependentes). Os participantes consideram o tema "muito relevante", pois na sua experiência, o cansaço e falta de tempo (com mais frequência do que o desejável) levam a cuidados mais técnicos e menos expressivos.

\section{2 ${ }^{a}$ Sessão: O que é o cuidado em enfermagem?}

As definições nos três contextos são similares, apresentando diferenças de ênfase (Quadro 2). Os enfermeiros de contexto hospitalar referem "mão", os de cuidados continuados indicam "mãos dadas" e os do centro de saúde "presença" (Foto 1). Com esta simbologia revelam algumas das formas mais comuns de definir a enfermagem, traduzindo apoio técnico associado a componente humana e de acompanhamento. Todos referem o "diálogo/comunicação" como elemento central do cuidado em enfermagem, que deve ocorrer entre enfermeiros e incluir o utente e sua família.

Foto 1: "Presença no cuidar.

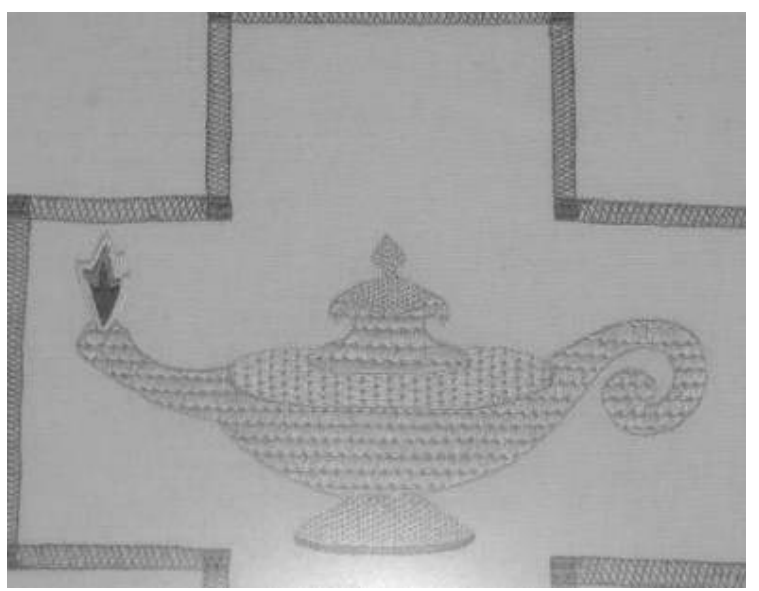

Não só a comunicação verbal, mas toda a comunicação analógica. (...) A nossa expressão facial e os nossos olhos muitas vezes falam! [Lúcia, 30 anos, cuidados domiciliários].

"Executar" e "estar disponível" são mencionados pelos enfermeiros do hospital e centro de saúde, mas não explicitamente pelos de cuidados continuados. "Estar disponível" refere-se a uma competência relacional, centrada na atenção ao outro e as suas necessidades. "Executar" centra a vertente mais técnica da enfermagem, relacionada com a execução dos cuidados, tais como, administração da medicação, puncionar e execução de pensos.

As técnicas têm que ser feitas. É algo que é exigido por nós e pelos colegas [Teresa, 26 anos, hospital].

"Humanizar" é explicitado pelos enfermeiros do hospital e dos cuidados continuados. Entendem que envolve respeitar o utente e estabelecer uma relação de proximidade/ajuda. Incluem os cuidados emocionais (pois, o equilibrio emocional é base para a satisfação de outras necessidades) e o desenvolvimento de uma relação terapêutica (de ajuda).

A relação de ajuda assenta sobre darmos-lhes várias hipóteses e eles decidem. Envolvê-los no processo de autonomia! [Maria, 22 anos, cuidados continuados].

Acima de tudo humanizar os cuidados é sermos humanos em relação a outra pessoa!" [Joana, 29 anos, hospital].

\section{$3^{a}$ Sessão: O que é ser idoso dependente?}

As pessoas idosas dependentes são consideradas pelos participantes como "necessitando de ..." (Quadro 2): i) em contexto hospitalar, ajuda total ou parcial; ii) nos cuidados primários apoio, pois como estão nas suas casas a ajuda é prestada mais por familiares, amigo e/ou vizinhos e os enfermeiros apoiam pela capacitação (ensinos) dos familiares, раra que possam prestar os cuidados adequados; iii) nos cuidados continuados, readaptação (Foto 2 ). Os participantes do hospital e do centro de saúde referem que as pessoas idosas dependentes são abandonadas, de alguma forma, pelos familiares.

Foto 2: "Reaprender.

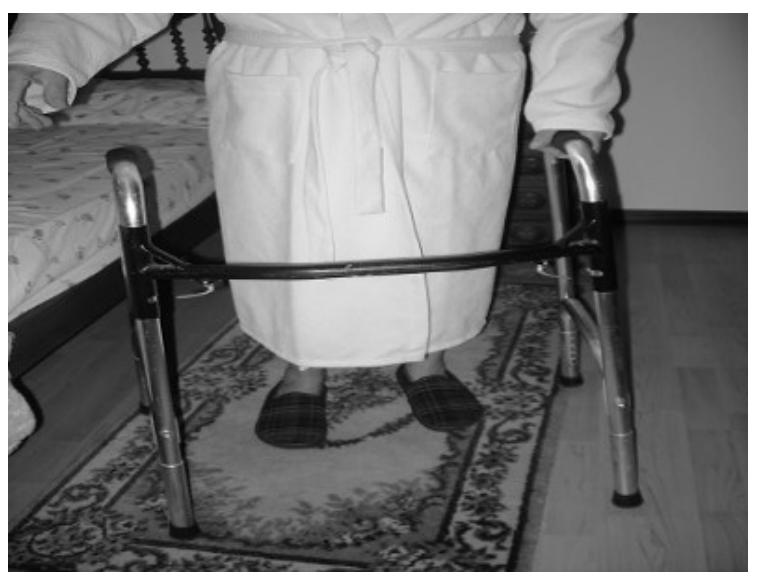


Os familiares não querem os idosos em casa e primeiro que a assistente social encontre um lar, os utentes estão no hospital mais uns dias! [Delfim, 43 anos, hospital].

Uma utente que está todo o dia sozinha, abandonada, a chorar porque tinha a fralda suja! [Carminda, 25 anos, cuidados domiciliares].

No contexto de centro de saúde, os enfermeiros afirmam que muitas vezes há abandono apesar de existir acompanhamento dos familiares. Muitas famílias, principalmente as mais vulneráveis, estão desprotegidas quando se trata de cuidar no seu domicílio do familiar dependente. Sublinham que até pode haver presença física ou acompanhamento, mas os doentes estão ali, à espera que "as senhoras do apoio ou as enfermeiras venham". Os participantes do hospital também salientam este abandono da família, que se evidencia quando tentam preparar a alta convocando os familiares e alguns não demonstram interesse. Consideram que talvez os familiares sintam medo dos cuidados que terão de prestar em casa, pois sentem escassa a aprendizagem a que têm acesso durante o internamento. Os participantes dos cuidados continuados não referem abandono.

$4^{a}$ Sessão: O que é o cuidado de enfermagem ideal a pessoas idosas dependentes?

Os resultados apontam para diferenças nos contextos (Quadro 2). Os enfermeiros do hospital mencionam "satisfazer as necessidades do doente", salientando a importância de englobar a família e outros significativos (Foto 3).

Foto 3: "Entreajuda".

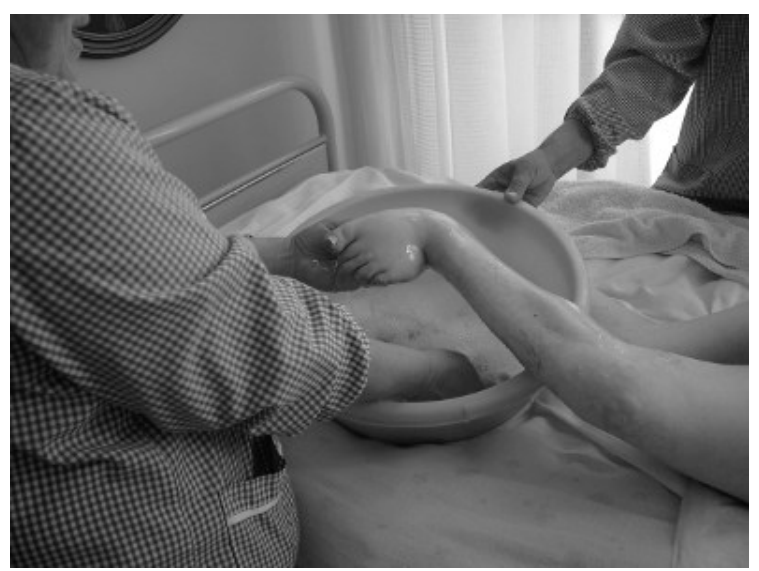

Os enfermeiros de centro de saúde referem as "parcerias" com o doente, sua família e rede informal, assim como com outros profissionais e instituições da comunidade, envolvidos numa relação horizontal. Neste sentido, sublinham a necessidade de uma equipe multidisciplinar (com diversas competências técnicas, humanas, éticas e científicas) com meios materiais e humanos para se deslocar até à pessoa idosa dependente e sua família, respondendo às suas necessidades. Salientam que a prestação de cuidados ideais só ocorrerá se houver envolvimento da família, caracterizada pelo "ideal humano" (boa relação entre o idoso e a sua família). Ou seja, a família deve gostar de ter a pessoa idosa dependente em casa, pois caso contrário não vale a pena estar em casa. Por isso, é necessário que a família revele em relação ao idoso: dedicação (complemento dos apoios formais), preocupação (querer o melhor) e esperança (de o ver melhor).

Os enfermeiros de cuidados continuados referem "integrar a família", centrando o envolvimento da família na continuidade dos cuidados, através da formação (ensinos); porque a preparação da família para o acolhimento no domicílio é essencial para a continuação e recuperação.

Considero que é um dos aspetos mais importantes. A preparação da família para o acolhimento em domicílio é fundamental para a continuação da recuperação do utente![Ana, 28 anos, cuidados continuados]

Assim, o cuidado às pessoas idosas difere consoante os contextos e é influenciado pelas especificidades de cada contexto.

$5^{a}$ Sessão: $O$ que é o cuidado de enfermagem real a pessoas idosas dependentes?

Os resultados sugerem diferenças entre os contextos (Quadro 2). Os enfermeiros do hospital mencionam "cuidados prestados no limite", pois asseguram os cuidados técnicos, mas nem sempre respondem aos de cariz mais humano; isto porque prestam cuidados a doentes idosos muito dependentes, num ambiente de ocupação total das camas do serviço.

Os enfermeiros do centro de saúde mencionam "cuidados parciais quotidianos", sublinhando que os cuidados de enfermagem reais são aqueles que efetuam 
da melhor forma que podem, nas condições que existem e no tempo disponível. Salientam que há falta de tempo para escutar e esclarecer dúvidas (garantem os cuidados técnicos, mas o resto fica comprometido porque lhes atribuem um número elevado de domicílios).

Os enfermeiros dos cuidados continuados referem que os cuidados ideais são "os que prestamos", pois dão prioridade à continuidade dos cuidados promovendo a autonomia, personalizando os cuidados e evitando as rotinas. Referem que talvez fossem necessários mais enfermeiros.

Os enfermeiros de cuidados hospitalares e domiciliares referem que os cuidados reais são efetuados sem o apoio da família. Mas, ambos os grupos de enfermeiros afirmam ter pouco tempo para dispensar aos familiares, pois o escasso tempo de que dispõem é para prestar cuidados técnicos ao doente (Foto 4), que são os exigidos pelos colegas, serviços e familiares.

Foto 4: "Executar técnicas".

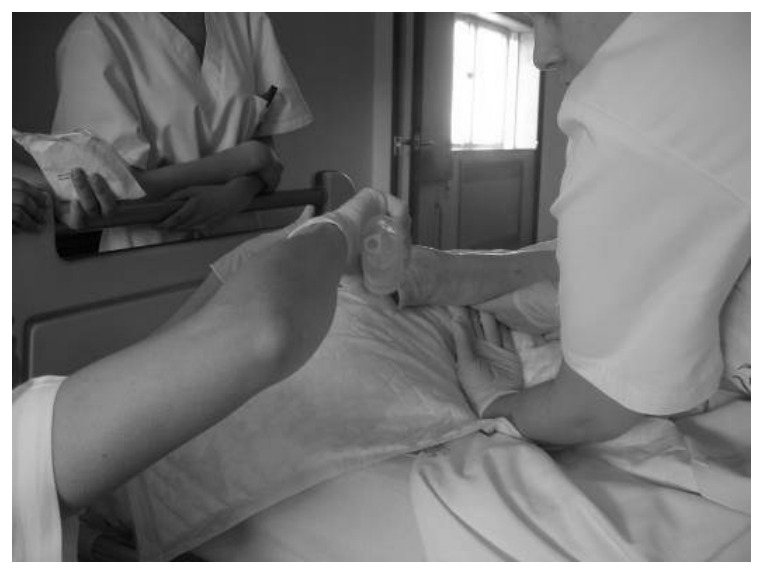

$6^{a}$ Sessão: O que afasta os cuidados reais do ideal?

Os enfermeiros consideram que os cuidados reais são afastados dos ideais devido a "escassez" (todos) e desorganização (hospital e centro de saúde); os enfermeiros de cuidados continuados salientam o "não envolvimento da família" (Quadro 2).

A escassez descreve cuidados prestados com um número de enfermeiros insuficiente, um número mais elevado daria tempo para cuidados de cariz humano (que valorizam) (Foto 5). Os enfermeiros de cuidados hospitalares e de centro de saúde referem ainda a escassez de material, espaço, falta de condições nas casas das pessoas onde prestam cuidados, que implicam a necessidade de improvisar. Acrescentam a desorganização da gestão, designadamente devido à frágil articulação com outros serviços ou colegas.

Foto 5: Falta de tempo.

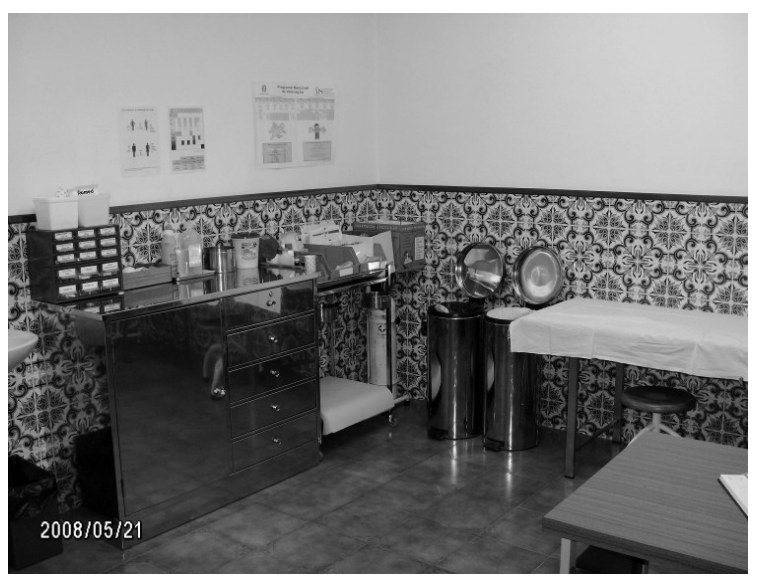

A estrutura é má mas consegue-se resolver os problemas desde que haja boa organização, boa metodologia. Por exemplo: vamos pedir 50 pensos porque estamos em contenção, sabendo que são precisos 100. Se faltar depois pede-se a outros serviços!" [Joana, 29 anos, hospital].

O não envolvimento da família é referido pelos enfermeiros de cuidados continuados, pois sem a família, mesmo que se prestem cuidados ideais de enfermagem, haverá sempre algo em falta: o apoio e compreensão das pessoas mais significativas.

$7^{a}$ Sessão: $O$ que facilitaria que o cuidado real se aproximasse do ideal?

Os participantes que trabalham em hospital e cuidados continuados apontam (Quadro 2): "trabalho e decisão em equipe multidisciplinar" (Foto 6) e salientam a necessidade de material. Os enfermeiros de centro de saúde acrescentam a necessidade de "manter o sonho". o trabalho e decisão em equipe multidisciplinar facilitariam a continuidade e consistência dos cuidados, assim como uma melhor tomada de decisão. A família é referida como parte integrante da equipa, pois os cuidados familiares (em geral, os informais) são essenciais e precisam de apoio. 
Foto 6: Decisão em equipe.

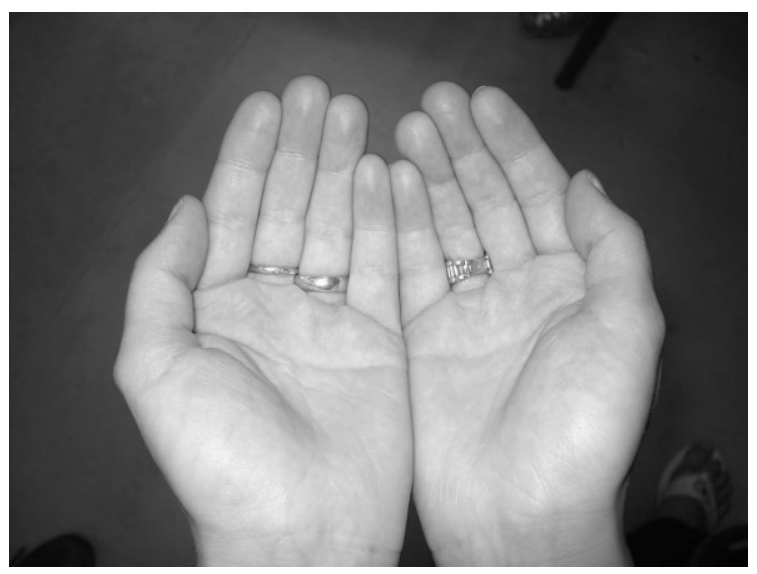

Apenas os participantes que trabalham em cuidados continuados afirmam ter uma equipa multidisciplinar mais coesa. Os participantes de cuidados domiciliares sublinham que os cuidadores informais são neste momento o principal parceiro de cuidados, por isso é necessário dotá-los de mais competências.

O facto de nos sentarmos à mesa e conversarmos sobre o que deveríamos fazer, quais as estratégias, faz-nos partilhar novas situações! [Lúcia, 30 anos, cuidados domiciliares]

Nos cuidados domiciliares a equipa é constituída por enfermeiro e família e só pontualmente por outro profissional, porque muitos centros de saúde apenas têm médicos, enfermeiros, auxiliares e administrativos. Por isso, os enfermeiros de centro de saúde salientam o seu lema: é necessário manter o sonho (vontade), acreditar, não desistir e dar sempre o melhor.

Os participantes que trabalham em hospital referem que uma equipa multidisciplinar organizada e articulada facilitaria a "existência e disponibilidade do material e equipamento" necessário para realizar e executar bons procedimentos. Além disso, permitiria melhor tomada de decisões, que muitas vezes são discutidas na passagem de turno apenas por elementos da equipa de enfermagem (sem a participação de outros profissionais de saúde). Ainda sublinham que a equipa multidisciplinar no hospital é alargada, pois para além de médicos e enfermeiros inclui fisioterapeutas, nutricionistas, assistentes sociais, psicólogos; contudo, só pontualmente é pedida colaboração destes outros profissionais de saúde.
Se tivermos bom equipamento, como no caso do desfibrilhador que agora temos, podemos prestar bons cuidados [Joana, 29 anos, hospital].

\section{DISCUSSÃO DOS RESULTADOS}

Os cuidados em enfermagem são definidos pelos participantes de forma similar para os três contextos, por meio da fotografia "mão" (hospital e cuidados continuados) e "presença" (centro de saúde). Estas imagens tradicionais em enfermagem são símbolo da capacidade evolutiva (fazer e transformar), representando um saber-fazer que procura responder à necessidade de alguém de forma humanizada. Paralelamente, consideram a pessoa idosa como alguém que necessita de ajuda (hospital), apoio (centro de saúde) ou readaptação (cuidados continuados), que são essencialmente prestados em termos técnicos. Ou seja, há alguma inconsistência no discurso: na definição dos cuidados de enfermagem valorizam a componente afetiva, embora reconhecendo que na prática assume um papel secundário face à técnica; caracterizam uma pessoa idosa internada pela "necessidade de ajuda" que especificam como precisar da execução de técnicas. Assim, a harmonia entre técnica e afetividade parece ainda um processo em desenvolvimento e mais fácil de descrever do que praticar.

O cuidado ideal à pessoas idosas dependentes apresenta algumas especificidades considerando o contexto: no hospital centra-se em satisfazer necessidades, uma vez que estes cuidados são dirigidos para a intervenção na crise, sendo preconizada a prestação de cuidados técnicos avançados; no centro de saúde focam as parcerias, pois envolvem uma vertente comunitária e incluem educação, promoção e prevenção da saúde; nos cuidados continuados centram-se em integrar a família, pois os utentes estão internados para recuperar e depois serem integrados na família e sociedade.

Os cuidados reais também apresentam especificidades contextuais: no hospital são cuidados prestados no limite; no centro de saúde são parciais; e em cuidados continuados são os que prestam (ou seja, próximos do ideal). Contudo, o que os afasta do ideal é similar, mesmo no caso dos cuidados continuados, e envolve escassez, desorganização e pouco apoio da família. 
A escassez de recursos e a desorganização tendem a traduzir-se em falta de tempo, desordem e frustração. $O$ ambiente social e organizacional condiciona a dinâmica de funcionamento, podendo mesmo retirar o doente do centro da ação. É importante que a filosofia institucional, assim como as políticas públicas de humanização, estejam atentas aos trabalhadores de saúde(15). A escassez de recursos (humanos e materiais) e a consequente desorganização desencadeiam um ambiente de trabalho onde impera a rapidez, agitação, cumprimento de tarefas, proporcionando o desenvolvimento de mecanismos de defesa nos enfermeiros que diminuem a sua sensibilidade ${ }^{(16-17)}$. As pessoas idosas dependentes requerem mais tempo de cuidados de enfermagem, o que raramente é considerado na organização dos serviços. A Ordem dos Enfermeiros $^{(18)}$ indica que, em 2007, existiam em Portugal 6,6 enfermeiros por mil habitantes, número aquém do rácio médio da UE: 7,5 por mil habitantes. Contudo a escassez de recursos nem sempre é impeditivo da prestação de cuidados de qualidade, pode mesmo ser geradora de formas inovadoras e criativas de resolução dos problemas ${ }^{(16)}$.

Os participantes referiram, também como obstáculo, à prestação de cuidados de enfermagem de qualidade, a falta de envolvimento familiar. Contudo, a investigação indica que o apoio a pessoas idosas está concentrado na família e que o abandono é mais um mito limitado a uma percentagem reduzida de pessoas idosas. Por exemplo, algumas famílias recusam-se a levar as pessoas idosas para casa após a alta hospitalar justificando não terem condições para as receber nos domicílios e a inexistência de vagas nas instituições.

Com frequência esta atitude é catalogada como desinteresse e abandono, mas pode ser muito realista, pois o aumento de pessoas idosas com dependência não tem sido acompanhado pelo desenvolvimento de estruturas sociais e de saúde. Acresce que as alterações estruturais e funcionais na família (tais como a integração da mulher no mercado de trabalho e o aumento das e/migrações) exigem que o apoio familiar seja combinado com o formal, isto é, a família que quer cuidar dos seus elementos idosos precisa de suporte de instituições e profissionais(16-17). Além disso, o cuidado às pessoas idosas emerge centrado no utente, a família não recebe cuidados, i.e. não é integrada na unidade de cuidado. Apenas se exige à família que tenha o familiar em casa e cuide dele, mas não se atende às suas necessidades, verificando-se muitas vezes sentimentos de revolta e tristeza por parte dos cuidadores informais. Num estudo acerca do cuidar da família a pessoas idosas dependentes por AVC, verificou-se que a origem de sentimentos negativos era atribuída aos profissionais de saúde ${ }^{(17)}$, que não estavam disponíveis para ensinar e colaborar com os familiares.

Os obstáculos que os enfermeiros tendem a identificar como inibidores da qualidade dos cuidados são extrínsecos a si, focando a falta de enfermeiros e de condições de trabalho ${ }^{(16-17)}$. Deste modo, os enfermeiros colocam-se numa postura de impotência adequada(19): não podem fazer mais nada, pois não depende deles. Seria importante refletirem sobre os obstáculos intrínsecos para poderem sair desta postura que é simultaneamente cômoda e frustrante.

Os enfermeiros envolvidos neste estudo destacaram que para promover a qualidade de cuidados de enfermagem a idosos é relevante o trabalho e decisão em equipa multidisciplinar (inclui o doente, sua família e rede informal). Uma equipa organizada e articulada facilitaria a prestação de cuidados as pessoas idosas. Destacam que a boa prestação de cuidados seria promovida se houvesse mais material, recursos humanos, melhores condições de trabalho, boas condições físicas e formação adequada.

A família faz parte da unidade de cuidado, mas não pode ser apenas um recurso, pois há que considerar o impacto da situação (doente dependente) na família. A equipa de saúde nem sempre se mostra acolhedora com a família, que pode estar a vivenciar um processo de intenso sofrimento ${ }^{(20)}$. Autores ${ }^{(21)}$ estudaram fatores que favorecem a participação do acompanhante no cuidado a idosos e verificaram que, na perspectiva dos cuidadores, a equipa de enfermagem poderia promover a participação do familiar por meio de apoio emocional e cognitivo, informações sobre quando e como participar nos cuidados e sobre as condições da doença e limitações do paciente. O envolvimento da família deve ocorrer em parceria, implicando a construção de uma boa relação entre o idoso, sua família e o profissional.

\section{CONCLUSÃO}

As pessoas idosas dependentes tendem a receber cuidados mais técnicos do que relacionais. Nos três contextos em estudo verifica-se que os cuidados 
prestados cumprem finalidades diferentes (embora complementares), contudo tendem a partilhar dificuldades similares para atingirem cuidados de maior qualidade. Os enfermeiros valorizam a componente humana do cuidar, mas reconhecem que a sua prática se centra na técnica. A maioria dos cuidados de enfermagem, dirigidos a pessoas idosas, centra a técnica e as rotinas, que os enfermeiros se esforçam por efetuar de forma humanizada. Tal ocorre essencialmente por falta de tempo e recursos humanos. Como meios facilitadores de uma boa prestação de cuidados referem trabalhar e decidir em equipa multidisciplinar, incluindo o envolvimento da família.

A prática de enfermagem pode ser repensada: i) a nível de gestão, considerando as necessidades na população idosa (designadamente os graus elevados de dependência que aumentam as necessidades de

\section{REFERÊNCIAS}

1. Parahyba MI, Simões, CS. A prevalência de incapacidade funcional em idosos no Brasil. Ciência e Saúde Colectiva. 2006;11(4):967-974.

2. Carvalhais M, Sousa L. Comportamentos dos enfermeiros e impacto em doentes idosos em situação de internamento hospitalar. Revista Eletrônica de Enfermagem. 2007;9(3):596-616.

3. Wilkerson F. Prática de Enfermagem nos Cuidados Domiciliáriosconceitos e aplicações: o utente idoso. $3^{\mathrm{a} e d}$. Robyn Rice Loures: Lusociência; 2004.

4. Giacomozzi CM, Lacerda MR. A prática da assistência domiciliar dos profissionais da estratégia de saúde da família. Texto \& Contexto Enfermagem. 2006; 15(004): 645-653.

5. Martins CA. Valorizar a Visitação Domiciliária. Revista Sinais Vitais. 2003;(48):8-16.

6. Dutra LS. Assistência pós alta hospitalar para pacientes com cuidados especiais. Boletim de Saúde. 2004;18(2): 213-223.

7. Lavinsky E, Vieira T. Processo de cuidar de idosos com acidente vascular encefálico: sentimentos dos familiares envolvidos. Acta Scientiarum. Health Sciences 2004;26(1): 67-84.

8. Caldas CP. Envelhecimento com dependência: responsabilidade e demandas da família. Saúde Pública. 2003;19(3):12-25.

9. Ministério da Saúde, Direcção Geral da Saúde. Plano Nacional da Saúde 2004-2010. Lisboa (Portugal); 2004. Available from: http://www.dgsaude.min-saude.pt/pns/media/pns vol1.pdf.

10. Ministério do Trabalho e da Solidariedade, Rede Nacional de Cuidados Integrados. Decreto-lei $n^{\circ} 101 / 2006,6$ de Junho, publicado em DR n 109, I série-A. Lisboa (Portugal); 2006. http://www.rncci.min-

saude.pt/SiteCollectionDocuments/38563865.pdf

11. Wang CC; Yi WK, Tao Z. Photovoice as a participatory health promotion strategy. Health Promotion International. 1998;13(1):7586.

12. Wang, CC. Photovoice: A participatory Action Research Strategy Applied to Women's Heath. 1999;8(2):185-192.

13. Garcia $C ;$ Medeiros M. Ar, água e terra: percepções de saúde ambiental de adolescentes de origem mexicana. Rev. Eletr. Enf. 2007;9(3):574-587.

14. Fortin M. O processo de investigação da concepção à realização. Loures: Ed. Lusociência; 1999.

15. Backes DS, Lunardi VL, Lunardi FD. A humanização hospitalar como expressão da ética. Revista Latino-Americana Enfermagem. 2006;14(1): 345-353. cuidados de enfermagem); ii) a nível da prática dos enfermeiros que terão de reforçar os cuidados expressivos, associando-os aos cuidados mais técnicos; iii) atendendo às necessidades das famílias que precisam de apoio e formação para poderem retornar a casa com o seu familiar idoso prestando os cuidados adequados.

Este estudo apresenta diversas limitações, nomeadamente, a dimensão reduzida da amostra, que decorreu do método de recolha de dados que causava receios nos participantes e thes ocuparia muito tempo. Contudo, o método é rico e pode desvanecer alguns limites. Como perspectivas de pesquisas futuras seria interessante ultrapassar estas limitações e recolher dados através de outros métodos para comparar e ouvir outros atores: pessoas idosas, seus familiares e outros profissionais.

16. Marin MJ, Angerami EL. Caracterização de um grupo de idosos hospitalares e seus cuidadores visando o cuidado pós alta hospitalar. Revista Escola Enfermagem USP. 2002;36(1):33-41.

17. Araújo IM. Cuidar das famílias com um idoso dependente por AVC: Do hospital à comunidade. Revista Referência. 2008; II (7):7889.

18. Ordem dos Enfermeiros. Saúde: dados estatísticas 2000-2011. Lisboa (Portugal); $2012 . \quad$ Available from: http://www.ordemenfermeiros.pt/membros/Paginas/DadosEstatisti cos.aspx.

19. Sousa L, Eusébio C. When multi-problem poor individuals' myths meet social services myths. Journal of Social Work. 2007;7(2):217237.

20. Silva L. O papel da solidariedade desempenhado por familiares visitantes e acompanhantes de adultos e idosos hospitalizados. Texto \& Contexto Enfermagem. 2008;17(2): 56-71.

21. Pena SB, Diogo MJ. Factores que favorecem a participação do acompanhante no cuidado do idoso hospitalizado. Revista Latino Americana de Enfermagem. 2005;3(5): 106-121.

Artigo recebido em 30/03/2011. Aprovado para publicação em 11/06/2012. Artigo publicado em 30/09/2012. 